\title{
NÚMERO CROMOSSÔMICO EM ESPÉCIES BRASILEIRAS DE ADESMIA DC. (LEGUMINOSAE-FABOIDEAE) ${ }^{1}$
}

\author{
Recebido em 5.12.91. Aceito em 11.07 .92
}

\author{
Silvia Teresinha Sfoggia Miotto ${ }^{2}$ \\ Eliana Regina Forni-Martins ${ }^{3}$
}

\begin{abstract}
RESUMO - (Número cromossômico em espécies brasileiras de Adesmia DC. (LeguminosaeFaboideae)). Foram determinados números cromossômicos para 11 espécies de Adesmia ocorrentes na região sul do Brasil. As preparações citológicas foram obtidas mediante o esmagamento de células de pontas de raízes pela técnica da orceína acética. O número cromossômico para todas as espécies estudadas foi $2 n=20$, excetuando-se $A$. incana var. incana, $\operatorname{com} 2 n=c a .40$. Os dados são inéditos para nove espécies e para as demais há concordância com a literatura. Sugere-se o número básico $\mathrm{x}=10$ para o gênero. Até o momento somente quatro espécies foram citadas como poliplóides.

Palavras-chaves: Adesmia, cromossomos, Leguminosae, Faboideae.

ABSTRACT - (Chromosome numbers in Brazilian species of Adesmia DC. (LeguminosaeFaboideae)). Chromosome numbers of 11 South-Brazilian species of Adesmia were determined. The cytological preparations were obtained by squashing cells of root tips, using the acetic-orcein method. The chromosome number for all the species studied was $2 n=20$, excepting $A$. incana var. incana with $2 \mathrm{n}=\mathrm{ca} .40$. The counts are new for nine species, and the other two agree with the literature. It is suggested $x=10$ as the basic number for the genus. Up to the present only four species were cited as polyploid.
\end{abstract}

Key words: Adesmia, chromosomes, Leguminosae, Faboideae.

\section{Introdução}

$\mathrm{O}$ gênero Adesmia $\mathrm{DC}$. pertence à tribo Adesmieae (Benth.) Hutch. Abrange cerca de 230 espécies, exclusivamente sulamericanas, agrupadas em dois subgêneros e 43 séries. No Brasil o gênero é exclusivo da Região Sul, onde ocorrem 17 espécies, incluídas em quatro séries do subgênero Adesmia. Muitas das espécies são consideradas potencialmente forrageiras e algumas estão sendo avaliadas agronomicamente (Miotto, 1991).

\footnotetext{
' Parte da tese de doutorado da primeira autora, apresentada no Curso de Biologia Vegetal da Unicamp.

2 Departamento de Botânica/UFRGS, Av. Paulo Gama s/n. ${ }^{\circ}$ 90046-900, Porto Alegre-RS, Brasil.

${ }^{3}$ Departamento de Botânica/IB/UNICAMP, Caixa Postal 6109, 13081-970, Campinas-SP, Brasil.
} 
O gênero Adesmia é, até o presente momento, pouco estudado do ponto de vista citogenético, dispondo-se na literatura de relatos de números cromossômicos para apenas 20 epítetos,correspondentes a 17 espécies válidas. O número cromossômico citado para a maioria das espécies é $2 \mathrm{n}=20$ (Castronovo 1945, Covas \& Schnack 1946, Covas 1949, Krapovickas \& Krapovickas 1951). ou n=10 (Rahn 1960, Hunziker et al. 1985). Também são referidos alguns casos de poliploidia, com $2 n=40$ cromossomos (Castronovo 1945, Covas 1949, Covas \& Hunziker 1954, Hunziker et al. 1985). Uma única citação de $2 \mathrm{n}=10$ é apresentada para $A$. aff. trijuga Gill. (Covas \& Schnack 1946).

O presente trabalho tem como objetivos contribuir para o conhecimento do número cromossômico de espécies brasileiras, fornecer subsídios para o estudo taxonômico do gênero e para futuros trabalhos de melhoramento genético de algumas de suas espécies.

\section{Material e métodos}

Foram determinados os números cromossômicos somáticos de 11 espécies, citadas na Tabela 1 , juntamente com o número de coleta e a procedência das sementes utilizadas. Materiais testemunhas estão depositados no Herbário CEN (Centro Nacional de Pesquisas de Recursos Genéticos e Biotecnologia, EMBRAPA, Brasília, DF) podendo haver duplicatas nos Herbários ICN (Departamento de Botânica, Instituto de Biociências, UFRGS, Porto Alegre, RS) e UEC (Departamento de Botânica, IB, UNICAMP, Campinas, SP).

Sementes escarificadas foram postas a germinar em placas de Petri, sobre uma camada de algodão, recoberta por papel de filtro, levemente umidecidos, à temperatura ambiente. As radículas emergidas foram coletadas ao atingirem 1,0 a $1,5 \mathrm{~cm}$ de comprimento e pré-tratadas com solução saturada de paradiclorobenzeno (PDB) por 3 a 5 horas, numa temperatura entre 16 e $18^{\circ} \mathrm{C}$. A seguir, as raízes foram fixadas em solução de álcool etílico e ácido acético glacial na proporção volumétrica de 3:1 e estocadas em congelador. Preparações citológicas foram obtidas pelo esmagamento de pontas de raízes, utilizando a técnica da orceína acética (Medina \& Conagin 1964) ligeiramente modificada; após hidrólise em orceína acética a 2\% e $\mathrm{HCl} 1 \mathrm{~N}(9: 1)$, por 30 minutos, a $40^{\circ} \mathrm{C}$, as raízes foram transferidas para orceína acética a $1 \%$ durante cerca de 10 minutos, corante que também foi utilizado na montagem das lâminas. Medições foram feitas diretamente nas fotomicrografias para estimar a ordem de grandeza dos cromossomos.

O número cromossômico somático de cada espécie foi obtido através da observação de diversas células, conforme indicado na Tabela 1. Outras espécies de Adesmia tiveram seu número cromossômico obtido na literatura, a título de comparação (Bolkhovskikh et al. 1969, Moor 1973, 1974, 1977, Goldblatt 1981b, 1984, 1985, 1988). 
Tabela 1. Espécies de Adesmia utilizadas no estudo citológico, com os respectivos material testemunha e procedência. $\mathrm{k}=$ número de células analisadas, $2 \mathrm{n}=$ número cromossômico diplóide, $\mathrm{x}=$ número básico, * $=$ contagem cromossômica inédita.

\begin{tabular}{|c|c|c|c|c|c|}
\hline Séries e Espécies & $\mathrm{k}$ & $\begin{array}{l}\text { Material- } \\
\text { Testemunha }\end{array}$ & Procedência & $2 n$ & $\begin{array}{l}\text { Nível } \\
\text { de ploidia }\end{array}$ \\
\hline \multicolumn{6}{|l|}{ Série $S U B N U D A E$} \\
\hline *A. securigerifolia Hert. & 27 & VALLS et al. 9615 & RS: Bagé & 20 & $2 \mathrm{x}$ \\
\hline *A. riograndensis Miotto & 25 & VALLS et al. 9590 & $\begin{array}{l}\text { RS: Santana } \\
\text { da Boa Vista }\end{array}$ & 20 & $2 x$ \\
\hline \multicolumn{6}{|l|}{ Série BICOLORES } \\
\hline A. bicolor (Poir.) DC. & 3 & VALLS et al. 9614 & RS: Bagé & 20 & $2 \mathrm{x}$ \\
\hline A. incana Vog. var. incana & 3 & VALLS et al. 9637 & $\begin{array}{l}\text { RS: Santana } \\
\text { do Livramento }\end{array}$ & ca. 40 & $4 \mathrm{x}$ \\
\hline \multicolumn{6}{|l|}{ Série PSORALEOIDES } \\
\hline *A. tristis Vog. & 7 & VALLS et al. 10814 & RS: Vacaria & 20 & $2 \mathrm{x}$ \\
\hline *A. rocinhensis Burk. & 5 & VALLS et al. 7470 & SC: São Joaquim & 20 & $2 \mathrm{x}$ \\
\hline \multirow[t]{2}{*}{ *A. psoraleoides Burk. } & 1 & VALLS et al. 11325 & PR: Guarapuava & 20 & $2 \mathrm{x}$ \\
\hline & 7 & VALLS et al. 8003 & $\begin{array}{l}\text { SC: Bom Jardim } \\
\text { da Serra }\end{array}$ & 20 & $2 x$ \\
\hline \multirow[t]{2}{*}{ *A. vallsii Miotto } & 7 & VALLS et al.11347 & PR: Guarapuava & 20 & $2 \mathrm{x}$ \\
\hline & i & VALLS et al.11439 & PR: Palmas & 20 & $2 \mathrm{x}$ \\
\hline *A. sulina Miotto & 5 & VALLS et al.11508 & PR: Palmas & 20 & $2 \mathrm{x}$ \\
\hline \multirow[t]{2}{*}{ *A. ciliata Vog. } & 3 & VALLS et al. 7949 & SC: São Joaquim & 20 & $2 \mathrm{x}$ \\
\hline & 4 & VALLS et al. 11515 & PR: Palmas & 20 & $2 x$ \\
\hline $\begin{aligned} & \text { Série MURICATAE } \\
& * \text { *. muricata (Jacq.)DC. }\end{aligned}$ & & & & & \\
\hline $\begin{array}{l}\text { *A. muricata (Jacq.)DC. } \\
\text { var. muricata }\end{array}$ & $\begin{array}{l}4 \\
16\end{array}$ & $\begin{array}{l}\text { VALLS et al. } 9570 \\
\text { VALLS \& SILVA }\end{array}$ & $\begin{array}{l}\text { RS: Canguçu } \\
\text { RS: Caçapava }\end{array}$ & $\begin{array}{l}20 \\
20\end{array}$ & $\begin{array}{l}2 x \\
2 x\end{array}$ \\
\hline & & 10289 & do Sul & & \\
\hline
\end{tabular}

\section{Resultados e discussão}

Os números cromossômicos obtidos para as 11 espécies aqui estudadas são apresentados na Tabela 1. Os cromossomos são pequenos, variando, em média, de 1,0 a $1,5 \mu \mathrm{m}$ de comprimento (Figura 1).

Os resultados de números cromossômicos são inéditos para nove das espécies estudadas: A. securigerifolia, A. riograndensis, A. tristis, A. rocinhensis, A. psoraleoides, A. vallsii, A. sulina, A. ciliata e A. muricata var. muricata. Para A. bicolor e A. incana os resultados coincidiram com os apresentados por Castronovo (1945) (Tabela 2). Adesmia incana var. incana foi o único táxon que apresentou número cromossômico diferente de $2 \mathrm{n}=20$, tendo sido observados cerca de 40 cromossomos.

Reunindo os dados aqui obtidos (Tabela 1) aos encontrados na literatura (Tabela 2), dispõe-se de relatos de números cromossômicos para 26 espécies, equivalendo a cerca de apenas $11 \%$ do gênero Adesmia (Miotto 1991).

Sugere-se que o número básico para o gênero seja $x=10$, concordando com a proposição de Castronovo (1945). A indicação de x=10 também é apoiada na consideração filogenética feita por Goldblatt (1981a), que considerou a tribo Adesmieae citologicamente ligada à tribo Aeschynomeneae, ambas com predominância de $\mathrm{n}=10$ cromossomos. 

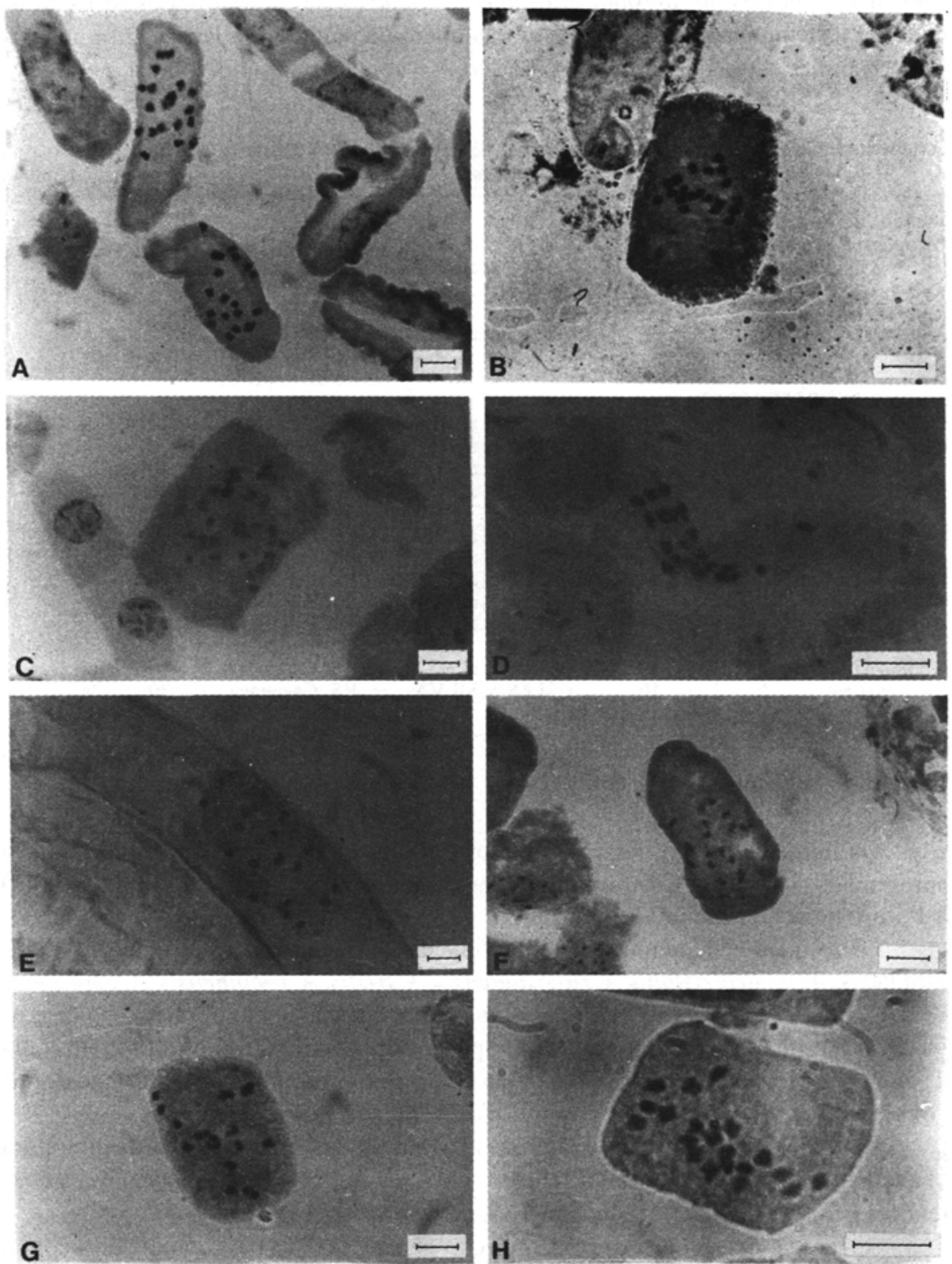

FIGURA 1 - Cromossomos mitóticos metafásicos em espécies de Adesmia. A - A. securigerifolia ( $2 \mathrm{n}=20)$. B - A. riograndensis $(2 \mathrm{n}=20)$. C - A. incana var. incana $(2 \mathrm{n}=\mathrm{ca} \cdot 40)$. D - A. tristis $(2 \mathrm{n}=20)$. E - A. vallsii $(2 n=20) . F-A$. sulina $(2 n=20) . G-A$. ciliata $(2 n=20) . H-A$. muricata var. muricata $(2 n=20)$. Barra $=5$ $\mu \mathrm{m}$. 
Tabela 2 . Números cromossômicos de espécies de Adesmia, segundo a literatura. * = espécies que ocorrem no Brasil; + = sinônimo de A. trijuga Hook. et Arn.; \# = sinônimo de A. corymbosa Clos. (Sinônimos segundo Burkart 1967). A grafia dos binômios é a dos trabalhos citados.

\begin{tabular}{lll}
\hline Séries e Espécies & Referências & N. ${ }^{\circ}$ cromos. \\
& $n$ Procedência
\end{tabular}

\section{SUBGÊNERO ACANTHADESMIA}

SÉRIE LONGISETAE

$\begin{array}{lllll}\text { \#A. fernandezii Phil. } & \text { Covas \& Schnack 1946 } & - & 20 & \text { Argentina } \\ \text { \#A. palenae } \text { Phil. } & \text { Rahn } 1960 & 10 & - & \text { Argentina }\end{array}$

\section{SÉRIE MICROPHYLLAE}

+ A. capricornu Phil.

Covas 1949

A. nov.spec.af.glanduligera Johnst.(sic) Covas 1949

A. obovata Clos.

A. pinifolia Gill.

Rahn 1960

Castronovo 1945

A. remyana Phil.

Krapovickas \&

Krapovickas 1951

A. shneideri Phil.

A. aff. trijuga Gill.

Rahn 1960

Castronovo 1945

Covas \& Schnack 1946

Castronovo 1945

Covas 1949

- 20 Argentina

- 20 e $40 \quad$ Argentina

$10-\quad$ Argentina

- 20

- 20

Argentina

Chile

A. trijuga Hook. et Arn.

A. uspallatensis Gill.ap.Hook.et Arn.

Covas 1949

$10-$

- 20 e 40

Argentina

- 10

- 20

Argentina

Argentina

Argentina

- 20

Argentina

Argentina

SÉRIE SUBTERRANEAE

A. subterranea Clos.

Krapovickas \&

Krapovickas 1951

$-20$

Chile

\section{SUBGÊNERO ADESMIA}

SÉRIE AMEGHINENSES

A. ameghinoi Speg.

Covas \& Hunziker 1954

- $38-40$

Argentina

SÉRIE BICOLORES

*A. bicolor (Poir.)DC.

*A. incana Vog.

A. incana Vog. var. grisea

(Hook.f.)Burk.

*A. punctata (Poir.)DC. var.

hilariana Benth

SÉRIE CORONILLOIDES

A. atuelensis Burk.

A. coronilloides Gill.

SÉRIE MACROSTACHYAE

A. macrostachya Benth.

\begin{tabular}{|c|c|c|c|}
\hline Castronovo 1945 & - & 20 & Argentina \\
\hline Castronovo 1945 & - & 40 & Argentina \\
\hline Hunziker et al. 1985 & - & 40 & Argentina \\
\hline Hunziker et al. 1985 & 10 & - & Argentina \\
\hline Bolkhovskikh et al. 1969 & - & 20 & ignorada \\
\hline Covas \& Schnack 1946 & - & 20 & Argentina \\
\hline Castronovo 1945 & - & 20 & Argentina \\
\hline
\end{tabular}

Entretanto, alguns autores indicaram $\mathrm{x}=5$ como o número básico para Adesmia (Covas \& Hunziker 1954; Hunziker et al. 1985), baseando-se numa única citação de 2n=10 para Adesmia aff. trijuga feita por Covas \& Schnack (1946). Estudando material aparentemente da mesma espécie e procedente da mesma localidade (Província de Mendoza, Puente del Inca), Castronovo (1945) obteve número cromossômico $2 \mathrm{n}=20$, portanto, diferente do apresentado por Covas \& Schnack (1946). Entretanto, 
devido à impossibilidade de comprovar a identificação botânica do material utilizado naqueles trabalhos, não se pode descartar a indicação de $x=5$. Novas contagens neste táxon poderão trazer informações adicionais para a confirmação do número básico do gênero.

A poliploidia parece ter desempenhado papel pouco importante na evolução do gênero, uma vez que foi observada em apenas quatro espécies (Tabela 2). Dentre as espécies brasileiras que tiveram seu número cromossômico determinado, a poliploidia foi constatada apenas em $A$. incana var. incana (Tabela 1).

A ocorrência de poliploidia não está relacionada com o arranjo taxonômico proposto para o gênero por Burkart (1967). As espécies até então relatadas como poliplóides pertencem a séries distintas, estando subordinadas a subgêneros diferentes. Ressalte-se ainda que, nas séries aonde ocorrem espécies poliplóides encontramse também espécies diplóides, sugerindo que a poliploidia apareceu independentemente em cada um destes grupos. Além disso, autores que mencionaram a ocorrência de poliploidia em $A$. aff. trijuga (Castronovo 1945) e em $A$. aff. glanduligera (Covas 1949) também apresentam a citação de $2 \mathrm{n}=20$ para estas mesmas espécies (Tabela 2).

Excluindo as espécies poliplóides já citadas, verifica-se uniformidade no número cromossômico das espécies de Adesmia. A constância do número cromossômico, o pequeno número de espécies estudadas citologicamente e a inexistência de estudos cariotípicos mais detalhados dificultam a utilização destes dados para uma interpretação evolutiva do gênero.

\section{Agradecimentos}

Ao CENARGEN-EMBRAPA, Brasília, DF e à EMPASC, Lages, SC, pela coleta e envio de sementes de diversas espécies utilizadas neste trabalho.

\section{Referências bibliográficas}

Bolkhovskikh, Z.; Grif, V.; Matvejeva, T. \& Zakharyeva, O. 1969. In: Fedorov, A. A. (ed.).Chromosome number of flowering plants. Moscou: V. L. Komarov Botanical Institute, Academy of Sciences of the U.S.S.R.

Burkart, A. 1967. Sinopsis del género sudamericano de Leguminosas - Adesmia DC. (Contribución al estudio del género Adesmia. VII). Darwiniana 14(2-3):463-568.

Castronovo, A. 1945. Estudio cariológico de doce especies de Leguminosas argentinas. Darwiniana 7(1): 38-57.

Covas, G. \& Schnack, B. 1946. Número de cromossomos en antófitas de la región de Cuyo (Republica Argentina). Rev. Arg. Agron. 13(3):153-166.

Covas, G. 1949. Estudios cariológicos en antófitas, III. Darwiniana 9(1):158-162.

Covas, G. \& Hunziker, J. H. 1954. Estudios cariológicos en antófitas. IV. Rev. Invest. Agric. 8(3):249-253.

Goldblatt, P. 1981 a. Cytology and the phylogeny of Leguminosae. In: Polhill, R. M. \& Raven, P. H. (eds.). Advances in Legume Systematics, vol. 2. Kew: Royal Botanic Gardens, p. 427-463.

Goldblatt, P. (ed.). 1981b. Index to plant chromosome numbers: 1975-1978. Monographs in Systematic Botany from the Missouri Botanical Garden, vol. 5.

Goldblatt, P. (ed.). 1984. Index to plant chromosome numbers: 1979-1981. Monographs in Systematic Botany from the Missouri Botanical Garden, vol. 8. 
Goldblatt, P. (ed.). 1985. Index to plant chromosome numbers: 1982-1983. Monographs in Systematic Botany from the Missouri Botanical Garden, vol. 13,

Goldblatt, P. (ed.). 1988. Index to plant chromosome numbers: 1984-1985. Monographs in Systematic Botany from the Missouri Botanical Garden, vol. 23.

Hunziker, J. H., Xifreda, C. C. \& Wulff, A. F. 1985. Estudios cromosomicos en angiospermas de Sudamerica. Darwiniana 26(1-4):7-14.

Krapovickas, A. \& Krapovickas, A. M. F. 1951. Notas citológicas sobre Leguminosas. Darwiniana 9(34):612-613

Medina, D. M. \& Conagin, C. H. T. M. 1964. Técnica citológica. Publicação n. ${ }^{\circ}$ 2610. Campinas: Instituto Agronômico.

Miotto, S. T. S. 1991. O gênero Adesmia DC. (Leguminosae - Faboideae) no Brasil. Campinas: Universidade Estadual de Campinas. Tese de Doutorado.

Moore, R. J. (ed.). 1973. Index to plant chromosome numbers. Regnum Vegetabile 90:1-539.

Moore, R. J. (ed.). 1974. Index to plant chromosome numbers. Regnum Vegetabile 91:1-108.

Moore, R. J. (ed.). 1977. Index to plant chromosome numbers. Regnum Vegetabile 96:1-257.

Rahn, K. 1960. Chromosome numbers in some South American angiosperms. Bot. Tidsskr. 56(2):121-122. 